\title{
Electrically tunable low-density superconductivity in a monolayer topological insulator
}

\author{
Valla Fatemi1*, Sanfeng Wu ${ }^{1 *+}$, Yuan Cao', Landry Bretheau ${ }^{2}$, Quinn D. Gibson ${ }^{3}$, Kenji Watanabe', \\ Takashi Taniguchi ${ }^{4}$, Robert J. Cava ${ }^{5}$, Pablo Jarillo-Herrero' ${ }^{1} \uparrow$ \\ ${ }^{1}$ Department of Physics, Massachusetts Institute of Technology, Cambridge, MA 02139, USA. ${ }^{2}$ Laboratoire des Solides Irradiés, École Polytechnique, CNRS, CEA, 91128 \\ Palaiseau Cedex, France. ${ }^{3}$ Department of Chemistry, University of Liverpool, Liverpool L69 7ZX. UK. ${ }^{4}$ Advanced Materials Laboratory, National Institute for Materials \\ Science, 1-1 Namiki, Tsukuba 305-0044, Japan. ${ }^{5}$ Department of Chemistry, Princeton University, Princeton, NJ 08544, USA. \\ *These authors contributed equally to this work. †Corresponding author. Email: swu02@mit.edu (S.W.); pjarillo@mit.edu (P.J.-H.)
}

Turning on superconductivity in a topologically non-trivial insulator may provide a route to search for nonAbelian topological states. However, existing demonstrations of superconductor-insulator switches have involved only topologically trivial systems. Here we report reversible, in situ electrostatic on/off switching of superconductivity in the recently established quantum spin Hall insulator monolayer tungsten ditelluride $\left(\mathrm{WTe}_{2}\right)$. Fabricated into a van der Waals field effect transistor, the monolayer's ground state can be continuously gate-tuned from the topological insulating to the superconducting state, with critical temperatures $T_{c}$ up to $\sim 1 \mathrm{Kelvin}$. Our results establish monolayer $\mathrm{WTe}_{2}$ as a material platform for engineering nanodevices that combine superconducting and topological phases of matter.

The intersection of superconductivity and topological insulators hosts a fertile landscape of interesting quantum phenomena, including non-Abelian topological excitations (1-3). Some topological insulators have been turned into superconductors via chemical doping $(4,5)$, application of pressure (6$8)$, or via the proximity effect $(9,10)$, methods that are either irreversible or ex situ. Proximity effect-based devices have in particular been engineered in an effort to realize Majorana physics; this has required fine-tuned interface engineering between distinct materials (11-16).

Here we report the observation of intrinsic superconductivity in the monolayer topological insulator $\mathrm{WTe}_{2}$ induced by the electric field effect. This monolayer transition metal dichalcogenide has recently been established as a quantum spin Hall insulator with robust edge transport up to $100 \mathrm{Kel}-$ vin (17-22). Our field effect device geometry (23) is illustrated in Fig. 1, A and B. The WTe $\mathrm{W}_{2}$ monolayer flake is van der Waalsencapsulated between two sheets of hexagonal boron nitride (BN) to protect it from chemical degradation and to improve transport characteristics. Top and bottom electrostatic gates, for which the same BN sheets serve as gate dielectrics, are fabricated to modulate the carrier density. An optical microscopy image of a typical device (device 1, BN thicknesses: $15 \mathrm{~nm}$, top; $8 \mathrm{~nm}$, bottom) is shown in Fig. 1B.

Figure 1C displays the temperature dependence of the four-probe resistance $R(T)$ in device 1 when the monolayer is n-doped $\left(V_{b g}=4 \mathrm{~V} ; V_{t g}=5 \mathrm{~V}\right) . R$ drops to zero at low temperatures from $1.2 \mathrm{k} \Omega$ in the normal state, and this zero-resistance state is present for a wide range of gate voltage parameters (Fig. 1C, inset). The DC voltage-current ( $V-I)$ characteristics at various temperatures (Fig. 1D) exhibit the transition from Ohmic behavior (red curve) at $\sim 1 \mathrm{~K}$ to highly nonlinear behavior (black curve) at low temperatures, including the characteristic zero voltage plateau for finite current, typical of superconductivity. This nonlinearity is further captured by the measurement shown in Fig. 1E, where the four-probe differential resistance, $d V / d I$, is plotted as a function of DC current bias $I_{D C}$ at base temperature. Under application of an out-of-plane magnetic field $B$, the nonlinear behavior is clearly suppressed (Fig. 1E), as expected for superconductivity. These observations confirm a superconducting state forming in the monolayer atomic sheet, in contrast to its 3D parent crystal, in which no superconductivity is found unless high pressure is applied $(24,25)$. We note that a small shoulder appears around $400 \mathrm{mK}$ in the $R(T)$ curve (Fig. 1C). Although this may be an intrinsic feature of $\mathrm{WTe}_{2}$, it could also result from the appearance of inhomogeneous superconducting islands as the temperature is reduced. We then characterize the superconducting transition by quantifying several important temperature scales. The onset of superconductivity, defined as the temperature at which $R$ drops to $90 \%$ from its normal state, occurs at $\sim 820 \mathrm{mK}$. Zero resistance is achieved at $\sim 350 \mathrm{mK}$. Furthermore, we have also confirmed the $2 \mathrm{D}$ nature of the superconductivity by checking that the data fit the picture of a 2D Berezinskii-Kosterlitz-Thouless transition, with estimated critical temperature $T_{B K T} \sim 470 \mathrm{mK}$ (fig. S1). For simplicity in our discussion, we define the critical transition temperature $T_{c}$ as the temperature at which $R$ equals $50 \%$ of the normal state value. For the curve in Fig. 1C, $T_{c} \sim 580 \mathrm{mK}$ as indicated. Similar behavior is observed in de- 
vice 2 , in which $T_{c}$ is found to be as high as $\sim 950 \mathrm{mK}$, although the resistance never drops to zero owing to a known imperfection in this device [figs. S2 and S3, (23)]. In contrast to device 1 , the $R(T)$ characteristic of device 2 is a good fit to the standard Aslamazov-Larkin and Maki-Thompson fluctuation conductivity terms $(26,27)$ (fig. S1).

The observed superconductivity, and hence the monolayer's ground state, is easily gate-tunable. We reveal this aspect by plotting $R(T)$ for different $V_{b g}$ (Fig. 2A), while $V_{t g}$ is kept fixed at $5 \mathrm{~V}$ (all data in Fig. 2 is for device 1). One can clearly see a critical gate voltage $V_{c}^{M I T} \sim-0.75 \mathrm{~V}$ at which a metal to insulator transition occurs. At large gate voltages $V_{b g}$ $>V_{c}^{M I T}$ (i.e., toward higher electron density), $R$ decreases with decreasing $T$, and a zero-resistance state is observed at low enough temperature, indicating a superconducting phase. Slightly above $V_{c}^{M I T}$, there may exist an intrinsic metallic (non-superconducting) phase at zero temperature, but a more detailed study is necessary to confirm this, as the observed behavior near the transition may also be consistent with the formation of both superconducting and non-superconducting regions as a result of density inhomogeneities. In contrast, when $V_{b g}<V_{c}^{M I T}$ (i.e., lower electron density), $R$ increases with decreasing $T$, pointing to an insulating ground state. This insulating behavior is consistent with the expectation for monolayer $\mathrm{WTe}_{2}$ at low density: a topologically nontrivial insulating state exists at low temperatures (19-21), and edge state conduction is known to be marginal for the measurement configuration of device 1 (19). Figure S2 shows a similar transition behavior for device 2 . Given that the field effect is applied through a moderate dielectric constant material $\left(\kappa_{B N} \sim 3.5\right)$, these observations demonstrate that the ground state of the monolayer can be easily tuned between the two extremes of electron transport in materials, i.e., from the insulating to the superconducting state. For comparison, similar field effect behavior in existing superconducting systems is achieved only by extreme charge density doping using ionic liquids (28), using ultrahigh- $\kappa$ dielectric materials such as $\mathrm{SrTiO}_{3}(29,30)$, or ferroelectric polarization (31).

The gate tunability of the superconducting state can be further characterized by the extraction of the critical temperature and measurements of the critical current. In the upper panel of Fig. 2B, we plot the extracted $T_{c}\left(V_{b g}\right)$, where one finds increasing $T_{c}$ with increasing $V_{b g}$. Extrapolating to $T_{c}=0 \mathrm{~K}$ (red curve, see also fig. S4), we find a critical gate voltage $V_{c 1} \sim-0.65 \mathrm{~V}$. The maximum observed $T_{c} \sim 0.61 \mathrm{~K}$ appears at the highest applicable gate voltages for this device. Figure $2 \mathrm{C}$ shows $d V / d I$ vs $I_{\mathrm{DC}}$ at $V_{b g}=V_{t g}=5 \mathrm{~V}$, where the sharp peak at the critical current $\left|I_{D C}\right|=I_{c}$ and the zero-resistance plateau for low currents demonstrate the expected behavior for superconductivity. The additional peak at $\left|I_{D C}\right|=I_{c}$ ' in this data set is consistent with the previously mentioned small shoulder in the $R(T)$ characteristic (Fig. 1C). The differential resistance is strongly modulated by gate voltage (Fig. 2D). The peaks at $\pm I_{c}$ monotonically shift toward zero bias with decreasing $V_{b g}$ and eventually merge into a single peak at zero bias in the insulating region. This modulation of $I_{c}$ is summarized in the lower panel of Fig. 2B, where we find that the critical gate voltage $V_{c 2}$, at which $I_{c}$ vanishes, falls in the range between $-0.6 \mathrm{~V}$ and $-0.8 \mathrm{~V}$.

Therefore, one finds that $V_{c 1}, V_{c 2}$, and $V_{c}^{M I T}$ are very close to each other, which identifies the critical gate voltage as $V_{b g}$ $=V_{c} \sim-0.7 \mathrm{~V}$ (when $V_{t g}=5 \mathrm{~V}$ ), above which superconductivity is exhibited. Using the capacitance model, we estimate the corresponding critical doping density as $n_{c} \sim 5 \times 10^{12} \mathrm{~cm}^{-2}(23)$. Hall effect measurements confirm a low absolute carrier density in the superconducting region that is consistent with the density estimates from the electrostatic capacitance model (Fig. 2, E and F, and details in fig. S5). The visible deviation appearing at lower doping in the plot may be related to inhomogeneous transport in the monolayer (e.g., the presence of conducting edge channels), which can introduce a minor factor to the measured Hall density. A similar critical density $\left(\sim 3 \times 10^{12} \mathrm{~cm}^{-2}\right)$ is found in device $2(23)$. These are among the lowest critical density values reported for 2D superconductors (32). Such low-density superconductivity in monolayer $\mathrm{WTe}_{2}$ is the key to its extreme gate tunability. We also note that the maximum $T_{\mathrm{c}}$ obtained here $(\sim 0.6 \mathrm{~K}$ for device 1 and $\sim 1 \mathrm{~K}$ for device 2 , at carrier density $\left.n_{2 D} \sim 1.8 \times 10^{13} \mathrm{~cm}^{-2}\right)$ is relatively high, compared to other $2 \mathrm{D}$ superconductors with similar density $(30,32)$.

The preliminary low temperature electronic phase diagram of monolayer $\mathrm{WTe}_{2}$ can now be summarized (Fig. 3A). Near zero charge density resides the quantum spin Hall insulator (QSHI) phase, robust up to $100 \mathrm{~K}$ (22). With n-type doping above the critical density, $n_{c}$, the superconducting ground state develops at temperatures below $T_{c}$ (superconductivity was not observed with p-type doping in the gate-accessible region). Above $T_{c}$, the system is metallic. The coexistence of QSHI and superconducting states in the same phase diagram establishes monolayer $\mathrm{WTe}_{2}$ as a material for observing physics at the intersection of topological insulating states and superconductivity. In Fig. 3B, we show data from device 2, in which superconductivity and the quantized QSH transport are observed in the same monolayer device. The helical edge transport in the QSHI phase in this device was characterized in detail in our previous report (22). Its superconducting behavior is characterized in fig. S3. These results therefore demonstrate the electrostatic field effect switching of superconductivity on and off in a QSHI system. Moreover, in the same device we also observed preliminary evidence that superconductivity can be introduced into the helical edge states by proximity effect via adjacent gate-induced superconducting regions (see details in fig. S6). In principle, such proximity-induced superconducting helical edge states can be used 
to construct devices hosting Majorana zero modes to study non-Abelian physics.

We further characterize the gate-tunable superconducting properties by examining the magnetic field dependence. As shown Fig. 4A, one finds an out-of-plane critical field at base temperature $B_{c 2, \perp} \sim 30 \mathrm{mT}$ (for $V_{t g}=V_{b g}=5 \mathrm{~V}$ ), at which half of the normal state resistance is recovered. The temperature and gate dependence of this critical field is summarized in fig. S7, which indicates that the Ginzburg-Landau coherence length is close to $100 \mathrm{~nm}$. This length is about an order of magnitude larger than the estimated transport mean free path (fig. S4), suggesting that the superconductivity is in the dirty limit. In contrast to the out-of-plane critical field, the in-plane critical field $B_{c 2}$,// is significantly larger: about $4.3 \mathrm{~T}$ is required to recover half the normal state resistance (Fig. $4 \mathrm{~B})$. This field is about 4 times the conventional Pauli paramagnetic limit, which is given by $1.84 T_{c} \sim 1.1 \mathrm{~T}$. We summarize the $B_{c 2}-T_{c}$ phase diagram for both the in-plane and outof-plane cases in Fig. 4C. We note that the crystal symmetry and band structure of monolayer $\mathrm{WTe}_{2}$ are very different from the $2 \mathrm{H}$-type transition metal dichalcogenides, in which Ising-type superconductivity is responsible for exceeding the Pauli limit (32). Other possible mechanisms for exceeding the Pauli limit include reduced electron g-factor, spin-triplet pairing, or strong spin-orbit scattering (33, 34). The superconductivity in the dirty limit is consistent with the spin-orbit scattering scenario $(33,34)$, in which we extract a spin-orbit scattering time $\sim 244$ fs [see fit in Fig. 4 and details in (23)]. However, we stress that our observations merit further study to understand the nature of the superconductivity and the exact mechanism for the large in-plane critical field in the $\mathrm{WTe}_{2}$ monolayer.

We comment now on several interesting directions to explore. One is to look for an optimal doping, e.g., to determine whether a superconducting dome exists in the phase diagram $(24,30,35)$. Another is to investigate the effects of the material's strong anisotropy (36) and tunable non-centrosymmetry $(17,37)$ on the superconductivity. A particularly interesting question is whether the observed superconductivity is topologically non-trivial. Although we currently do not have an answer, our results point to an exciting possibility of creating a 2D-crystal based topological superconductor using the proximity effect. Indeed, using local gates to achieve lateral modulation of superconducting and QSHI regions would enable the fabrication of tunable superconductor-QSHIsuperconductor topological Josephson junctions (fig. S6) and the investigation of Majorana modes in a single material. In addition, van der Waals heterostructures that interface monolayer $\mathrm{WTe}_{2}$ with materials such as the recently discovered 2D layered ferromagnets $(38,39)$ can be developed for studying the interplay between superconductivity, magnetism, and topology.

\section{REFERENCES AND NOTES}

1. L. Fu, C. L. Kane, Superconducting proximity effect and Majorana fermions at the surface of a topological insulator. Phys. Rev. Lett. 100, 096407 (2008). doi:10.1103/PhysRevLett.100.096407 Medline

2. M. Z. Hasan, C. L. Kane, Colloquium: Topological insulators. Rev. Mod. Phys. 82, 3045-3067 (2010). doi:10.1103/RevModPhys.82.3045

3. X.-L. Qi, S.-C. Zhang, Topological insulators and superconductors. Rev. Mod. Phys. 83, 1057-1110 (2011). doi:10.1103/RevModPhys.83.1057

4. Y. S. Hor, A. J. Williams, J. G. Checkelsky, P. Roushan, J. Seo, Q. Xu, H. W. Zandbergen, A. Yazdani, N. P. Ong, R. J. Cava, Superconductivity in $\mathrm{Cu}_{x} \mathrm{Bi}_{2} \mathrm{Se}_{3}$ and its implications for pairing in the undoped topological insulator. Phys. Rev. Lett 104, 057001 (2010). doi:10.1103/PhysRevLett.104.057001 Medline

5. L. A. Wray, S.-Y. Xu, Y. Xia, Y. S. Hor, D. Qian, A. V. Fedorov, H. Lin, A. Bansil, R. J. Cava, M. Z. Hasan, Observation of topological order in a superconducting doped topological insulator. Nat. Phys. 6, 855-859 (2010). doi:10.1038/nphys1762

6. M. Einaga, Y. Tanabe, A. Nakayama, A. Ohmura, F. Ishikawa, Y. Yamada, New superconducting phase of $\mathrm{Bi}_{2} \mathrm{Te}_{3}$ under pressure above $11 \mathrm{GPa}$. J. Phys. Conf. Ser. 215, 012036 (2010). doi:10.1088/1742-6596/215/1/012036

7. J. L. Zhang, S. J. Zhang, H. M. Weng, W. Zhang, L. X. Yang, Q. Q. Liu, S. M. Feng, X. C. Wang, R. C. Yu, L. Z. Cao, L. Wang, W. G. Yang, H. Z. Liu, W. Y. Zhao, S. C. Zhang, X. Dai, Z. Fang, C. Q. Jin, Pressure-induced superconductivity in topological parent compound $\mathrm{Bi}_{2} \mathrm{Te}_{3}$. Proc. Natl. Acad. Sci. U.S.A. 108, 24-28 (2011). doi:10.1073/pnas.1014085108 Medline

8. P. P. Kong, J. L. Zhang, S. J. Zhang, J. Zhu, Q. Q. Liu, R. C. Yu, Z. Fang, C. Q. Jin, W. G. Yang, X. H. Yu, J. L. Zhu, Y. S. Zhao, Superconductivity of the topological insulator $\mathrm{Bi}_{2} \mathrm{Se}_{3}$ at high pressure. J. Phys. Condens. Matter 25, 362204 (2013). doi:10.1088/0953-8984/25/36/362204 Medline

9. B. Sacépé, J. B. Oostinga, J. Li, A. Ubaldini, N. J. G. Couto, E. Giannini, A. F. Morpurgo, Gate-tuned normal and superconducting transport at the surface of a topological insulator. Nat. Commun. 2, 575 (2011). doi:10.1038/ncomms1586 Medline

10. S. Hart, H. Ren, T. Wagner, P. Leubner, M. Mühlbauer, C. Brüne, H. Buhmann, L. W. Molenkamp, A. Yacoby, Induced superconductivity in the quantum spin Hall edge. Nat. Phys. 10, 638-643 (2014). doi:10.1038/nphys3036

11. V. Mourik, K. Zuo, S. M. Frolov, S. R. Plissard, E. P. A. M. Bakkers, L. P. Kouwenhoven, Signatures of Majorana fermions in hybrid superconductorsemiconductor nanowire devices. Science 336, 1003-1007 (2012) doi:10.1126/science.1222360 Medline

12. S. Nadj-Perge, I. K. Drozdov, J. Li, H. Chen, S. Jeon, J. Seo, A. H. MacDonald, B. A. Bernevig, A. Yazdani, Observation of Majorana fermions in ferromagnetic atomic chains on a superconductor. Science 346, 602-607 (2014). doi:10.1126/science.1259327 Medline

13. W. Chang, S. M. Albrecht, T. S. Jespersen, F. Kuemmeth, P. Krogstrup, J. Nygård, C. M. Marcus, Hard gap in epitaxial semiconductor-superconductor nanowires. Nat. Nanotechnol. 10, 232-236 (2015). doi:10.1038/nnano.2014.306 Medline

14. H.-H. Sun, K.-W. Zhang, L.-H. Hu, C. Li, G.-Y. Wang, H.-Y. Ma, Z.-A. Xu, C.-L. Gao, D.-D. Guan, Y.-Y. Li, C. Liu, D. Qian, Y. Zhou, L. Fu, S.-C. Li, F.-C. Zhang, J.-F. Jia, Majorana zero mode detected with spin selective Andreev reflection in the vortex of a topological superconductor. Phys. Rev. Lett. 116, 257003 (2016). doi:10.1103/PhysRevLett.116.257003 Medline

15. E. Bocquillon, R. S. Deacon, J. Wiedenmann, P. Leubner, T. M. Klapwijk, C. Brüne, K. Ishibashi, H. Buhmann, L. W. Molenkamp, Gapless Andreev bound states in the quantum spin Hall insulator HgTe. Nat. Nanotechnol. 12, 137-143 (2017). doi:10.1038/nnano.2016.159 Medline

16. Q. L. He, L. Pan, A. L. Stern, E. C. Burks, X. Che, G. Yin, J. Wang, B. Lian, Q. Zhou, E. S. Choi, K. Murata, X. Kou, Z. Chen, T. Nie, Q. Shao, Y. Fan, S.-C. Zhang, K. Liu, J. Xia, K. L. Wang, Chiral Majorana fermion modes in a quantum anomalous Hall insulator-superconductor structure. Science 357, 294-299 (2017). doi:10.1126/science.aag2792 Medline

17. X. Qian, J. Liu, L. Fu, J. Li, Quantum spin Hall effect in two-dimensional transition metal dichalcogenides. Science 346, 1344-1347 (2014). doi:10.1126/science.1256815 Medline

18. F. Zheng, C. Cai, S. Ge, X. Zhang, X. Liu, H. Lu, Y. Zhang, J. Qiu, T. Taniguchi, K. Watanabe, S. Jia, J. Qi, J.-H. Chen, D. Sun, J. Feng, On the quantum spin Hall gap of monolayer $1 \mathrm{~T}^{\prime}-\mathrm{WTe}_{2}$. Adv. Mater. 28, 4845-4851 (2016). doi:10.1002/adma.201600100 Medline 
19. Z. Fei, T. Palomaki, S. Wu, W. Zhao, X. Cai, B. Sun, P. Nguyen, J. Finney, X. Xu, D. H. Cobden, Edge conduction in monolayer WTe2. Nat. Phys. 13, 677-682 (2017). doi:10.1038/nphys4091

20. S. Tang, C. Zhang, D. Wong, Z. Pedramrazi, H.-Z. Tsai, C. Jia, B. Moritz, M. Claassen, H. Ryu, S. Kahn, J. Jiang, H. Yan, M. Hashimoto, D. Lu, R. G. Moore, C.C. Hwang, C. Hwang, Z. Hussain, Y. Chen, M. M. Ugeda, Z. Liu, X. Xie, T. P. Devereaux, M. F. Crommie, S.-K. Mo, Z.-X. Shen, Quantum spin Hall state in monolayer 1T'-WTe2. Nat. Phys. 13, 683-687 (2017). doi:10.1038/nphys4174

21. Z.-Y. Jia, Y.-H. Song, X.-B. Li, K. Ran, P. Lu, H.-J. Zheng, X.-Y. Zhu, Z.-Q. Shi, J. Sun, J. Wen, D. Xing, S.-C. Li, Direct visualization of a two-dimensional topological insulator in the single-layer 1T'-WTe2. Phys. Rev. B 96, 041108 (2017). doi:10.1103/PhysRevB.96.041108

22. S. Wu, V. Fatemi, Q. D. Gibson, K. Watanabe, T. Taniguchi, R. J. Cava, P. JarilloHerrero, Observation of the quantum spin Hall effect up to 100 kelvin in a monolayer crystal. Science 359, 76-79 (2018). doi:10.1126/science.aan6003 Medline

23. See supplementary materials.

24. X.-C. Pan, X. Chen, H. Liu, Y. Feng, Z. Wei, Y. Zhou, Z. Chi, L. Pi, F. Yen, F. Song, X. Wan, Z. Yang, B. Wang, G. Wang, Y. Zhang, Pressure-driven dome-shaped superconductivity and electronic structural evolution in tungsten ditelluride. Nat. Commun. 6, 7805 (2015). doi:10.1038/ncomms8805 Medline

25. D. Kang, Y. Zhou, W. Yi, C. Yang, J. Guo, Y. Shi, S. Zhang, Z. Wang, C. Zhang, S. Jiang, A. Li, K. Yang, Q. Wu, G. Zhang, L. Sun, Z. Zhao, Superconductivity emerging from a suppressed large magnetoresistant state in tungsten ditelluride. Nat. Commun. 6, 7804 (2015). doi:10.1038/ncomms8804 Medline

26. V. V. Dorin, R. A. Klemm, A. A. Varlamov, A. I. Buzdin, D. V. Livanov, Fluctuation conductivity of layered superconductors in a perpendicular magnetic field. Phys. Rev. B Condens. Matter 48, 12951-12965 (1993). doi:10.1103/PhysRevB.48.12951 Medline

27. A. I. Larkin, A. A. Varlamov, Theory of Fluctuations in Superconductors (International Series of Monographs on Physics, Oxford Univ. Press, 2005).

28. Y. Saito, T. Nojima, Y. Iwasa, Gate-induced superconductivity in two-dimensional atomic crystals. Supercond. Sci. Technol. 29, 093001 (2016). doi:10.1088/09532048/29/9/093001

29. K. A. Parendo, K. H. S. B. Tan, A. Bhattacharya, M. Eblen-Zayas, N. E. Staley, A. M. Goldman, Electrostatic tuning of the superconductor-insulator transition in two dimensions. Phys. Rev. Lett. 94, 197004 (2005). doi:10.1103/PhysRevLett.94.197004 Medline

30. A. D. Caviglia, S. Gariglio, N. Reyren, D. Jaccard, T. Schneider, M. Gabay, S. Thiel, G. Hammerl, J. Mannhart, J.-M. Triscone, Electric field control of the $\mathrm{LaAlO}_{3} / \mathrm{SrTiO}_{3}$ interface ground state. Nature 456, 624-627 (2008). doi:10.1038/nature07576 Medline

31. C. H. Ahn, S. Gariglio, P. Paruch, T. Tybell, L. Antognazza, J. Triscone, Electrostatic modulation of superconductivity in ultrathin $\mathrm{GdBa}_{2} \mathrm{Cu}_{3} \mathrm{O}_{7-x}$ films. Science 284 , 1152-1155 (1999). doi:10.1126/science.284.5417.1152 Medline

32. Y. Saito, T. Nojima, Y. Iwasa, Highly crystalline 2D superconductors. Nat. Rev. Mater. 2, 16094 (2016). doi:10.1038/natrevmats.2016.94

33. R. A. Klemm, A. Luther, M. R. Beasley, Theory of the upper critical field in layered superconductors. Phys. Rev. B 12, 877-891 (1975). doi:10.1103/PhysRevB.12.877

34. M. Tinkham, Introduction to Superconductivity (Dover, 2004).

35. J. T. Ye, Y. J. Zhang, R. Akashi, M. S. Bahramy, R. Arita, Y. Iwasa, Superconducting dome in a gate-tuned band insulator. Science 338, 1193-1196 (2012). doi:10.1126/science. 1228006 Medline

36. L. R. Thoutam, Y. L. Wang, Z. L. Xiao, S. Das, A. Luican-Mayer, R. Divan, G. W. Crabtree, W. K. Kwok, Temperature-dependent three-dimensional anisotropy of the magnetoresistance in WTe2. Phys. Rev. Lett. 115, 046602 (2015). doi:10.1103/PhysRevLett.115.046602 Medline

37. E. Bauer, M. Sigrist, Eds., Non-Centrosymmetric Superconductors (Lecture Notes in Physics Series, Springer, 2012), vol. 847.

38. B. Huang, G. Clark, E. Navarro-Moratalla, D. R. Klein, R. Cheng, K. L. Seyler, D. Zhong, E. Schmidgall, M. A. McGuire, D. H. Cobden, W. Yao, D. Xiao, P. JarilloHerrero, X. Xu, Layer-dependent ferromagnetism in a van der Waals crystal down to the monolayer limit. Nature 546, 270-273(2017). doi:10.1038/nature22391 Medline

39. C. Gong, L. Li, Z. Li, H. Ji, A. Stern, Y. Xia, T. Cao, W. Bao, C. Wang, Y. Wang, Z. Q. Qiu, R. J. Cava, S. G. Louie, J. Xia, X. Zhang, Discovery of intrinsic ferromagnetism in two-dimensional van der Waals crystals. Nature 546, 265-269 (2017). doi:10.1038/nature22060 Medline

40. S. Wu, Replication data for: Electrically tunable low-density superconductivity in a monolayer topological insulator. Harvard Dataverse (2018); https://doi.org/10.7910/DVN/UFGFY2.

41. M. N. Ali, L. Schoop, J. Xiong, S. Flynn, Q. Gibson, M. Hirschberger, N. P. Ong, R. J. Cava, Correlation of crystal quality and extreme magnetoresistance of WTe2. EPL Europhys. Lett. 110, 67002 (2015). doi:10.1209/0295-5075/110/67002

42. L. Bretheau, J. I.-J. Wang, R. Pisoni, K. Watanabe, T. Taniguchi, P. Jarillo-Herrero, Tunnelling spectroscopy of Andreev states in graphene. Nat. Phys. 13, 756-760 (2017). doi:10.1038/nphys4110

43. L. G. Aslamasov, A. I. Larkin, The influence of fluctuation pairing of electrons on the conductivity of normal metal. Phys. Lett. A 26, 238-239 (1968). doi:10.1016/0375-9601(68)90623-3

44. K. Maki, The critical fluctuation of the order parameter in type-Il superconductors. Prog. Theor. Phys. 39, 897-906 (1968). doi:10.1143/PTP.39.897

45. R. S. Thompson, Microwave, flux flow, and fluctuation resistance of dirty type-II superconductors. Phys. Rev. B 1, 327-333 (1970). doi:10.1103/PhysRevB.1.327

46. X. Xi, Z. Wang, W. Zhao, J.-H. Park, K. T. Law, H. Berger, L. Forró, J. Shan, K. F. Mak, Ising pairing in superconducting $\mathrm{NbSe}_{2}$ atomic layers. Nat. Phys. 12, 139-143 (2016). doi:10.1038/nphys3538

47. S. C. de la Barrera, M. R. Sinko, D. P. Gopalan, N. Sivadas, K. L. Seyler, K. Watanabe, T. Taniguchi, A. W. Tsen, X. Xu, D. Xiao, B. M. Hunt, Tuning Ising superconductivity with layer and spin-orbit coupling in two-dimensional transition-metal dichalcogenides. Nat. Commun. 9, 1427 (2018). doi:10.1038/s41467-018-038884 Medline

\section{ACKNOWLEDGMENTS}

We thank Liang Fu for helpful discussions and Joel I-Jan Wang for assistance in operating the dilution refrigerator. Funding: This work was partly supported through AFOSR Grant No. FA9550-16-1-0382 as well as the Gordon and Betty Moore Foundation's EPiQS Initiative through Grant No. GBMF4541 to P. J-H. Device nanofabrication was partly supported by the Center for Excitonics, an Energy Frontier Research Center funded by the DOE, Basic Energy Sciences Office, under Award No. DE-SC0001088. This work made use of the Materials Research Science and Engineering Center's Shared Experimental Facilities supported by NSF under Award No. DMR-0819762. Sample fabrication was performed partly at the Harvard Center for Nanoscale Science supported by the NSF under Grant No. ECS-0335765. S.W. acknowledges the support of the MIT Pappalardo Fellowship in Physics. The WTe 2 crystal growth performed at Princeton University was supported by an NSF MRSEC grant, DMR-1420541. Growth of BN crystals was supported by the Elemental Strategy Initiative conducted by the MEXT, Japan and JSPS KAKENHI Grant Numbers JP15K21722 and JP25106006. Author contributions: V.F. and S.W. fabricated the devices, performed electronic transport measurements and analyzed the data. Y.C. and L.B. helped with measurements. Q.D.G. and R.J.C. grew the WTe $e_{2}$ crystals. K.W. and T.T. grew the BN crystals. P.J-H. supervised the project. V.F., S.W., and P.J$\mathrm{H}$. co-wrote the manuscript with input from all co-authors. Competing interests: The authors declare no competing financial interests. Data and materials availability: Hexagonal boron nitride crystals were provided by Kenji Watanabe and Takashi Taniguchi under a material transfer agreement with NIMS. All data needed to evaluate the conclusions in the paper are present in the paper or the supplementary materials and have been deposited at (40).

\section{SUPPLEMENTARY MATERIALS}

www.sciencemag.org/cgi/content/full/science.aar4642/DC1

Materials and Methods

Supplementary Text

Figs. S1 to S7

References (41-47)

12 November 2017; accepted 9 October 2018

Published online 25 October 2018

10.1126/science.aar4642 
A

A Monolayer $\mathrm{WTe}_{2}$

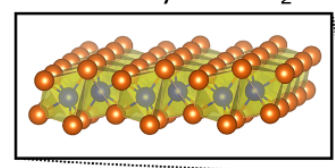

w $\odot$ Te

Contact

$\mathrm{BN}$

Bottom Gate

C

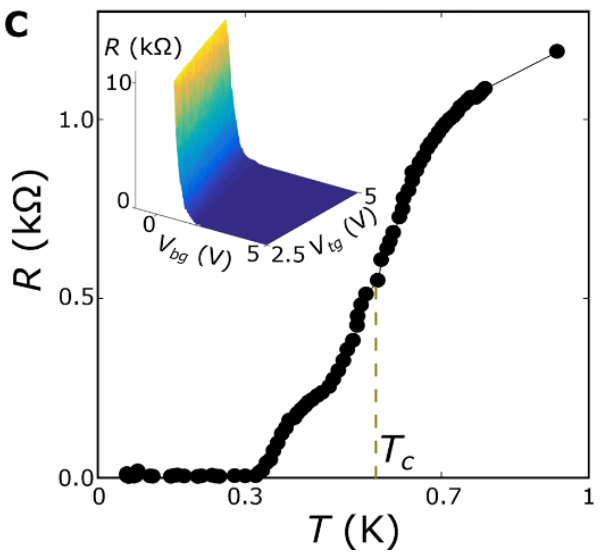

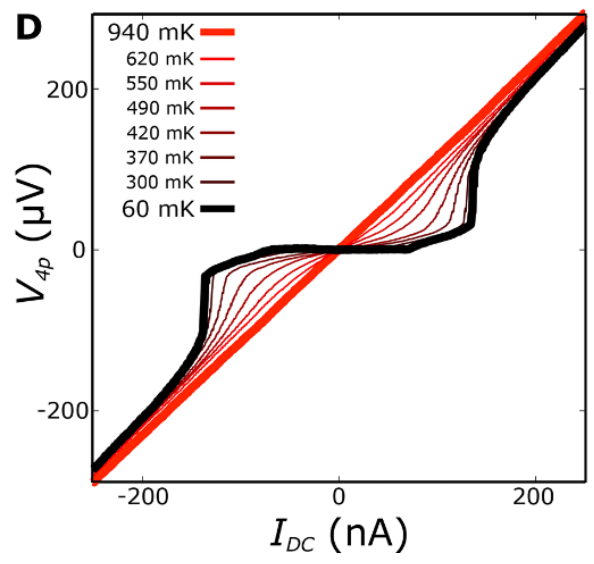

B
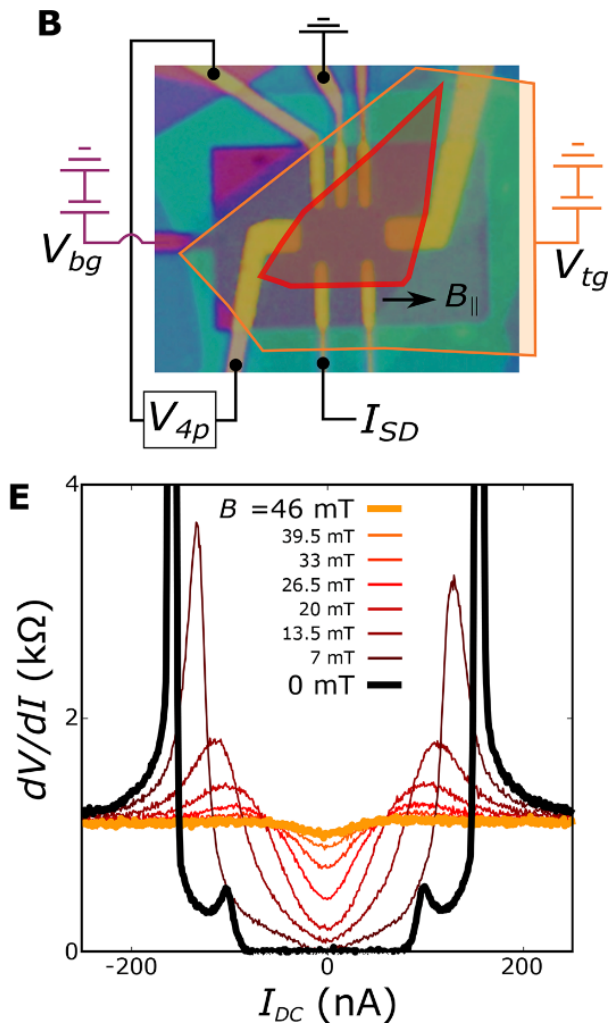

Fig. 1. Device schematic and superconductivity characteristics. (A) Cartoon illustration of the device structure and the crystal structure of monolayer WTe 2 . (B) Optical microscopy image of device 1, with the monolayer WTe 2 (red) and graphite top gate (orange) highlighted. Circuit elements show the measurement configuration. (C) Temperature dependence of the resistance for $V_{b g}=4 \mathrm{~V}$ and $V_{t g}=5 \mathrm{~V}$. Inset: the resistance as a function of both gate voltages, at base temperature of $60 \mathrm{mK}$. (D) $V$-I characteristics from base temperature (black) up to $940 \mathrm{mK}$ (red). (E) Nonlinear $V$-I behavior, captured by differential resistance curves, at base temperature for different perpendicular magnetic fields. 

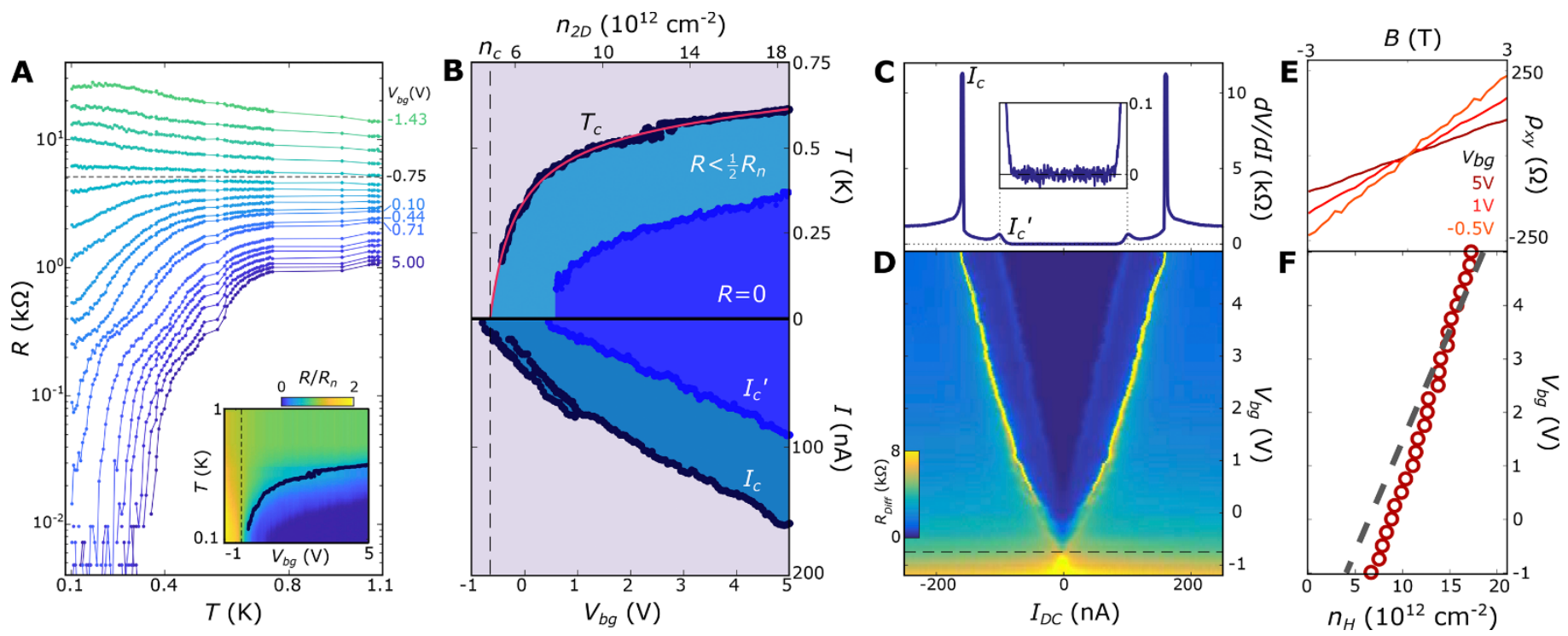

Fig. 2. Switching on/off superconductivity with an electrostatic gate. Shown are data from device 1. (A) $R(T)$ characteristic for different gate voltages, showing the transition from a superconducting state to an insulating state, in evenly spaced increments between the labeled curves. Dashed line is a guide to the gate voltage, $V_{c}{ }^{M I T}$, that separates the two regimes. Inset: color plot of the same data, normalized to the normal state resistance $R_{n}$, with $T_{c}$ marked in black. (B) Upper panel: gate dependent critical temperature $T_{\mathrm{c}}$, summarized from (A). The zero-resistance region is shaded dark blue. Lower panel: gate dependent critical current, $I_{c}$ and $I_{c}{ }^{\prime}$, summarized from (D). For $V_{b g}<1 \mathrm{~V}, I_{c}$ is found to bifurcate into two peaks, the values of which are determined by extrema of the second derivative of $d V / d I\left(I_{D C}\right)$ data. The electron density $\left(n_{2 D}\right)$, corresponding to bottom gate voltage shown on the bottom axis, is estimated from the capacitance model and shown on the top axis. (C) Differential resistance $d V / d l$ V.s. current bias $I_{D C}$ for $V_{t g}=V_{b g}=5 \mathrm{~V}$. Inset: zoom-in to the zero-resistance region. (D) Differential resistance $d V / d l$ vs. current bias $I_{D C}$ and gate voltage $V_{b g}$. Close to the $V_{b g} \sim 1$, the observed $I_{c}$ trace bifurcates, as more clearly indicated in (B). Dashed line indicates the gate voltage at which the peaks in differential resistance merge at zero bias, indicating destruction of superconductivity. (E) Hall resistivity $\rho_{x y}(B)$ for three selected bottom gate voltages taken at $2 \mathrm{~K}\left(V_{t g}=5 \mathrm{~V}\right)$. See details in (23). (F) Extracted Hall density as a function of the gate voltage (red circles) and the estimated density from the capacitance model (grey dashed line). 

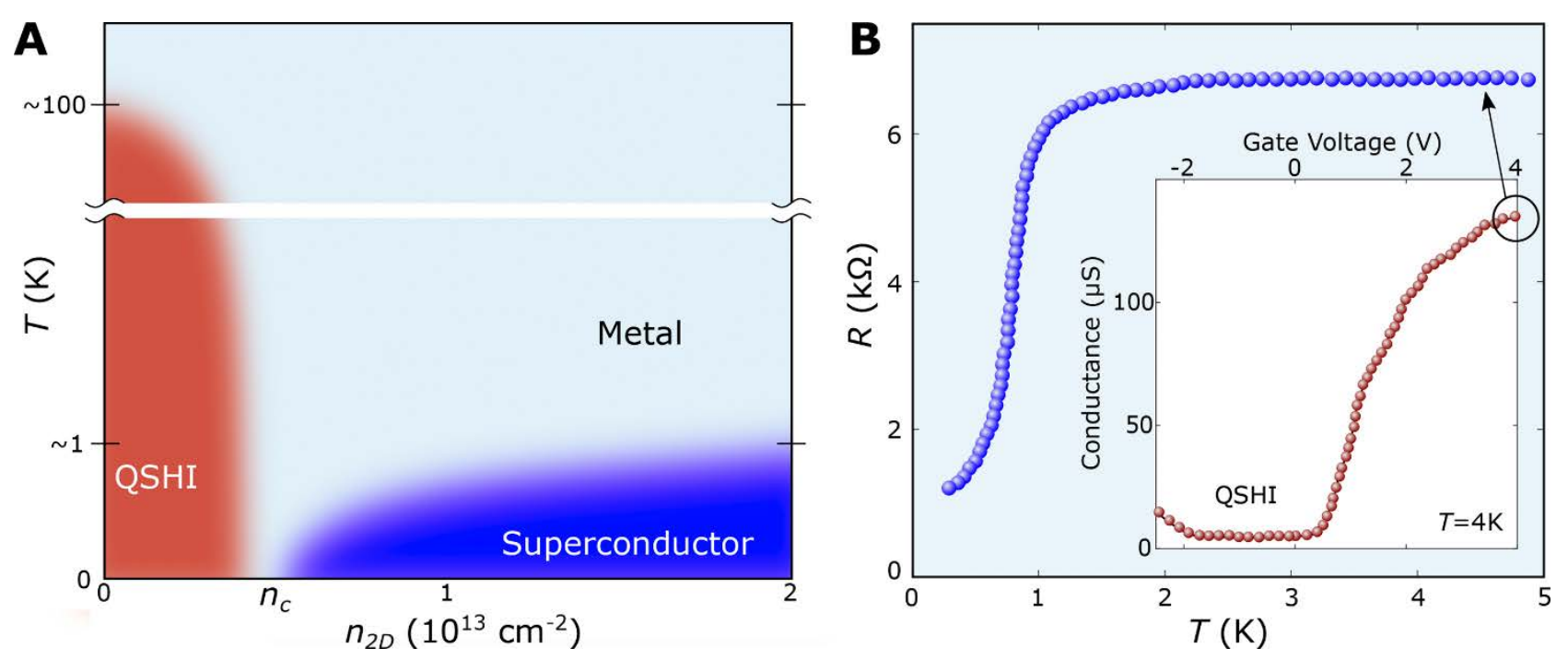

Fig. 3. Electronic phase diagram of monolayer WTe 2 . (A) Schematic temperature-density phase diagram for the electronic ground state of monolayer $\mathrm{WTe}_{2}$, reminiscent of the inset of Fig. 2A. (B) Resistance vs. temperature for device 2, which shows a clear superconducting transition. The residual resistance at low temperature is caused by a known imperfection in this device, as described in (23). Inset: Gate-dependent conductance of device 2, where the observed plateau corresponds to the QSHI phase. The arrow indicates the gate voltage at which the temperature dependence was recorded.
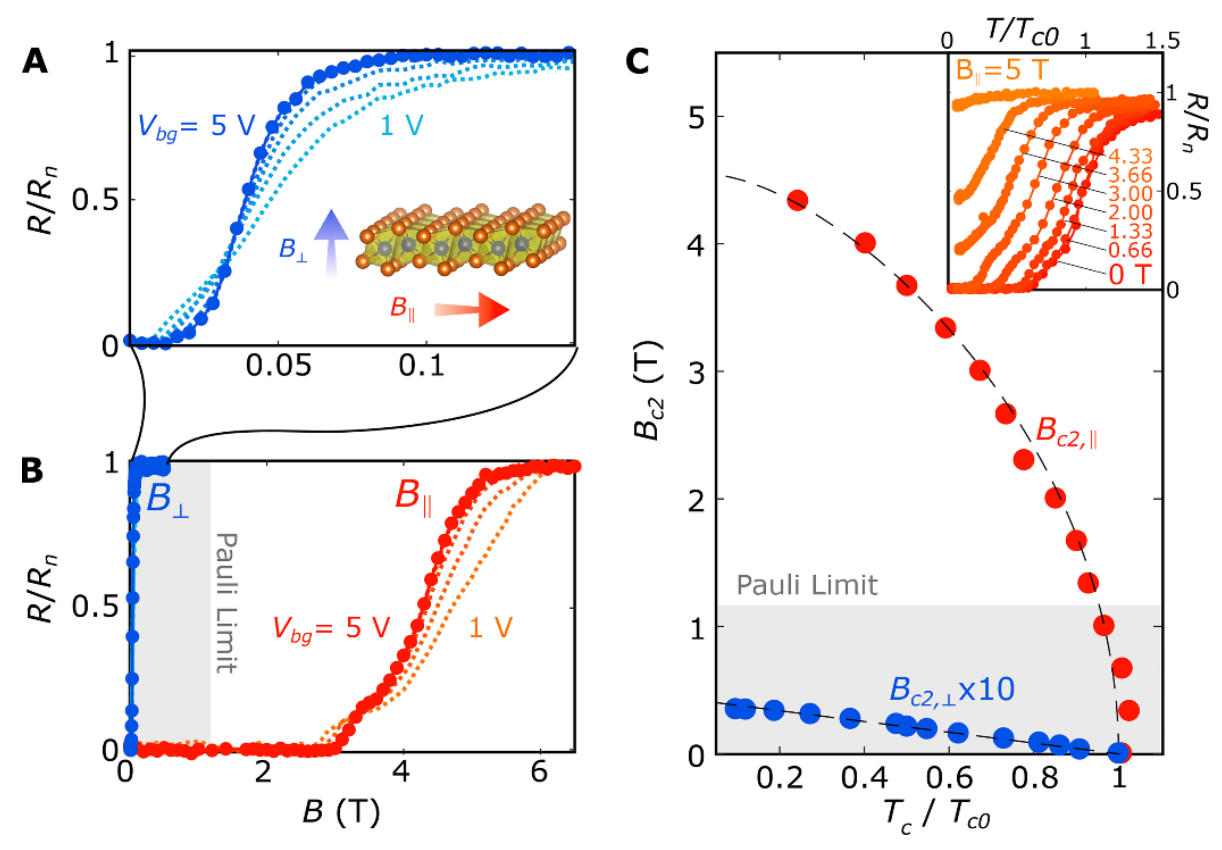

Fig. 4. Magnetic field effect on the monolayer superconductivity. Shown are data from device 1. (A) Perpendicular magnetic field dependence of the resistance, $R\left(B_{\perp}\right)$, normalized to the normal state resistance $R_{n}$, at base temperature for different gate voltages: $V_{t g}=5 \mathrm{~V}$ and $V_{b g}=5,4,3,2,1 \mathrm{~V}$. Inset: schematic of the perpendicular and parallel field orientations. See Fig. 1B for orientation of parallel magnetic field in the plane of the device. (B) Orange: parallel magnetic field dependence of resistance, $R\left(B_{\|}\right)$, normalized to the normal state resistance $R_{n}$, for the same series of gate voltages as in (A). Blue: the perpendicular magnetic field data of $(A)$ plotted on the same scale for comparison. (C) $B_{c 2}-T_{c}$ phase diagram for both the parallel (orange) and perpendicular (blue) orientations. $V_{t g}=$ $V_{b g}=5 \mathrm{~V}$. Black dashed lines are fits to theoretical models (23). Inset: the temperature dependence of resistance under different parallel magnetic fields. 